\title{
Association between Altered Blood Parameters and Gut Microbiota after Synbiotic Intake in Healthy, Elderly Korean Women
}

\author{
Song Hee Lee ${ }^{1,2}$, Hee Sang You ${ }^{1,2}$, Hee-Gyoo Kang ${ }^{2,3} \mathbb{D}$, Sang Sun Kang ${ }^{4}(\mathbb{D}$ \\ and Sung Hee Hyun 1,2,*(D) \\ 1 Department of Biomedical Laboratory Science, School of Medicine, Eulji University, 77 Gyeryong-ro, \\ 771 Beon-gil, Jung-gu, Daejeon 34824, Korea; song-1107@naver.com (S.H.L.); yhs1532@nate.com (H.S.Y.) \\ 2 Department of Senior Healthcare, BK21 Plus Program, Graduate School, Eulji University, 77 Gyeryong-ro, \\ 771 Beon-gil, Jung-gu, Daejeon 34824, Korea; kanghg@eulji.ac.kr \\ 3 Department of Biomedical Laboratory Science, College of Health Sciences, Eulji University, \\ Seongnam 13135, Korea \\ 4 Department of Biology Education, Chungbuk National University, Chungdae-ro 1, Seowon-gu, \\ Cheongju 28644, Korea; jin95324@cbu.ac.kr \\ * Correspondence: hyunsh@eulji.ac.kr; Tel.: +82-10-9412-8853
}

Received: 25 August 2020; Accepted: 9 October 2020; Published: 12 October 2020

\begin{abstract}
Synbiotics intake can alter the composition of intestinal microbes beneficially. We aimed to detect the changes in the intestinal microbiomes of 37 healthy elderly Korean women after the intake of a synbiotic drink. This was a longitudinal study controlled with a temporal series, including a control period of 3 weeks before intake, synbiotic intake for 3 weeks, and a washout period of 3 weeks. Fecal microbiota composition was analyzed by sequencing the V3-V4 hypervariable regions of $16 \mathrm{~S}$ rRNA. Physical fecal activity increased with improvement in fecal shape. Thirty intestinal bacterial taxa were observed to change only after the intake period. In particular, Ellagibacter appeared only after ingestion. In addition, the abundance of Terrisporobacter showed a positive correlation with C-reactive protein, triglyceride. Lachnospiraceae_uc, Eubacterium_g5, and Blautia had a positive correlation with creatinine, whereas PAC001100_g had a negative correlation with creatinine. Short-term (3 weeks) intake of symbiotic organisms changes the composition of the gut microbiota in healthy elderly Korean women.
\end{abstract}

Keywords: blood markers; Blautia; elderly women; Ellagibacter; $16 \mathrm{~S}$ rRNA; synbiotic drink

\section{Introduction}

The life expectancy of Koreans is 83.2 years, but the healthy lifespan (i.e., one without serious illness or injury) was only 64.4 years in 2018 [1,2]. Therefore, the current challenge is to maintain "active longevity" by extending the healthy lifespan and reducing the duration of illness. The human intestinal microbiota play an important role in maintaining gastrointestinal homeostasis and are beneficial for host health [3]. Further, dysbiosis led to an imbalance of beneficial and harmful microorganisms [3]. Gut microbiota dysbiosis causes, and is associated with, several diseases, ranging from localized gastrointestinal disorders (including constipation) and metabolic alterations, to respiratory, liver, cardiovascular, and psychiatric neurological disorders [4]. Using probiotics has been proposed as a potential therapy to restore the balance of healthy human intestinal function and gut bacterial composition [3]. According to the official definition of the World Health Organization (WHO), probiotics are "live microorganisms, which when consumed in adequate amounts, confer a healthy effect on the host" [5]. 
Although previous studies have showed the positive effects of probiotic consumption on several health outcomes, many of these studies focused on populations with specific pathologies [6-8], potentially resulting in side effects [9]. Evidence supporting the health-promoting effects of probiotics in healthy adults is limited and less consistent [10-12]. Although many studies have investigated correlations with specific blood markers by targeting a single strain to observe disease associations, the findings of these studies might result from numerous confounding factors [13]. There are also differences in microbial strains, viable cell concentrations, and probiotic product formulations [14], and most studies only controlled some factors affected by these variables. However, a clinical study requires the consideration of all potentially unknown variables. Studies targeting gut microorganisms displaying changes only upon the consumption of target food items are rare [15]. Thus, the aim of this study was to address this limitation and investigate the association between the gut microbiota and blood parameters upon synbiotic consumption.

Recent studies have investigated synbiotics, which are a mixture of one or more probiotics and one or more prebiotics that benefit the host by promoting the survival and colonization of live microbes in the gastrointestinal tract [16-19]. Synbiotics were developed to overcome the challenges of viability of probiotics; the reason for their use is based on the observed improvements in the number of viable probiotic bacteria during passage through the upper intestine [20].

There are some studies demonstrating the effectiveness of synbiotics [13,21-23]. Several studies have shown that the use of synbiotics has superior benefits over probiotics or prebiotics alone. In one study, it was observed that synbiotic intervention results in a significantly different fecal flow bacterial community compared to prebiotic or probiotic alone intervention [21]. In addition, it was much more effective than using probiotics in the satisfaction of bowel habits and overall relief of IBS symptoms [24] and showed an additive effect on constipation symptoms [25]. In animal studies, the use of synbiotics has shown significant anti-inflammatory effects in reducing disease severity, colon damage, and inflammatory mediators, while modulating intestinal metabolites and short chain fatty acids (SCFA) profiles. It has also been found that direct human testing is necessary [26].

The major effects of synbiotics include increasing beneficial gut microbiota and balancing gut microbiota [21-23,27,28]. However, no information is available on their impact on the entire phylogenetic structure of the intestinal microbiota in aged people. Therefore, this study observed the beneficial effects of synbiotic intake in healthy elderly women. Further, previous studies on the association between synbiotics and human health have mostly focused on Western populations, with few studies based on the Korean population [29]. In this study, 37 healthy elderly women were given a synbiotic drink for 3 weeks, and various blood and urine parameters were assessed along with fasting blood glucose levels. Furthermore, defecation activity was investigated using a questionnaire and the stool consistency was evaluated under the Bristol stool form scale (BSFS) [30,31]. Fecal microbiota were analyzed through next-generation sequencing of $16 \mathrm{~S}$ rRNA genes, and the association between the parameters described and the relative abundance and composition of microorganisms was investigated.

\section{Materials and Methods}

\subsection{Participants and Study Design}

This study included 39 healthy women volunteers selected among members of the Miraeseum Seongnam senior complex in Seongnam city, Gyeonggi-do Province, Korea. All participants provided written informed consent and were given the option to withdraw from the study at any time. The protocol was approved by the internal review board (IRB) of Eulji University (IRB No. EUIRB 2019-53). The inclusion criteria were healthy post-menopausal women over 50 (Table 1). The exclusion criteria were: chronic smokers ( $\geq 20$ cigarettes/day), alcoholism and those who consume $420 \mathrm{~g}$ alcohol per week, liver disease, kidney disease, cardiovascular disease, cerebrovascular disease, pancreatitis, cancer, thyroid disease, dementia, Parkinson's disease, depression, anorexia/bulimia, or multiple sclerosis. Participants were made aware that during the study, those who participate in other clinical 
research experiments or ingest probiotics, fermented products, and dietary supplements that affect body fat metabolism and intestinal health would be excluded from the study. The participants had to be willing to maintain a usual diet and physical activity levels and were asked to report concomitant medication, adverse effects, or any other comments. We conducted a survey consisting of questions such as family history, past history, and usual diet about the disease (Supplementary Materials Table S1).

Table 1. Clinical characteristics at baseline of the 37 subjects involved in the study.

\begin{tabular}{|c|c|c|c|}
\hline \multicolumn{2}{|c|}{ Variables } & \multirow[t]{2}{*}{$N$} & \multirow[t]{2}{*}{ Mean (SD) or Frequency (\%) } \\
\hline Age (Years) & & & \\
\hline & $50-59$ & 11 & $57.9(1.2)$ \\
\hline & $60-69$ & 15 & $63.3(3.2)$ \\
\hline & $70-79$ & 11 & $73.5(3.0)$ \\
\hline & Total & 37 & $65(6.7)$ \\
\hline \multirow[t]{2}{*}{ Menopausal transition } & Menstruating & 0 & 0 \\
\hline & Post-Menopausal & 37 & $100 \%$ \\
\hline \multirow[t]{3}{*}{ Smoking } & No (Non-smoking) & 37 & $100 \%$ \\
\hline & Yes (Smoking) & 0 & 0 \\
\hline & Ex-smoker & 0 & 0 \\
\hline \multirow[t]{2}{*}{ Alcohol } & No & 29 & $78 \%$ \\
\hline & Yes & 8 & $22 \%$ \\
\hline \multirow[t]{3}{*}{ Alcohol frequency of consumption } & Daily & 5 & \\
\hline & Weekly & 1 & \\
\hline & Occasionally & 2 & \\
\hline
\end{tabular}

The study was designed as a longitudinal, panel, pilot study and was controlled with a temporal series (Figure 1). The study included (1) a 3-week control period in which all participants maintained their usual diet and dietary habits, including restrictions on intake of probiotics, prebiotics, and synbiotics, followed by (2) a 3-week experimental period with the consumption of the synbiotic drink (one bottle and two capsules/day), and (3) a 3-week washout period without synbiotic drink intake. Anthropometric measurements and blood, urine, and fecal samples were collected. All participants responded to questionnaires related to basic information such as disease history, use of antibiotics, defecation, and diet. Necessary arrangements were made so the subjects would receive the synbiotic drink every week. The synbiotic was taken after meals to minimize the breakdown of probiotics by gastric acid [32]. Compliance with synbiotic drink consumption guidelines at home was monitored once per week through telephonic interviews. The primary objective of this study was to observe changes in the composition and abundance of gut microbiota, including Bifidobacterium and Lactobacillus, in elderly Korean women upon consumption of synbiotic drinks. The secondary objective was to observe the association between the blood parameters measured in this study and the bacteria that changed only upon ingestion of the synbiotic drink. 


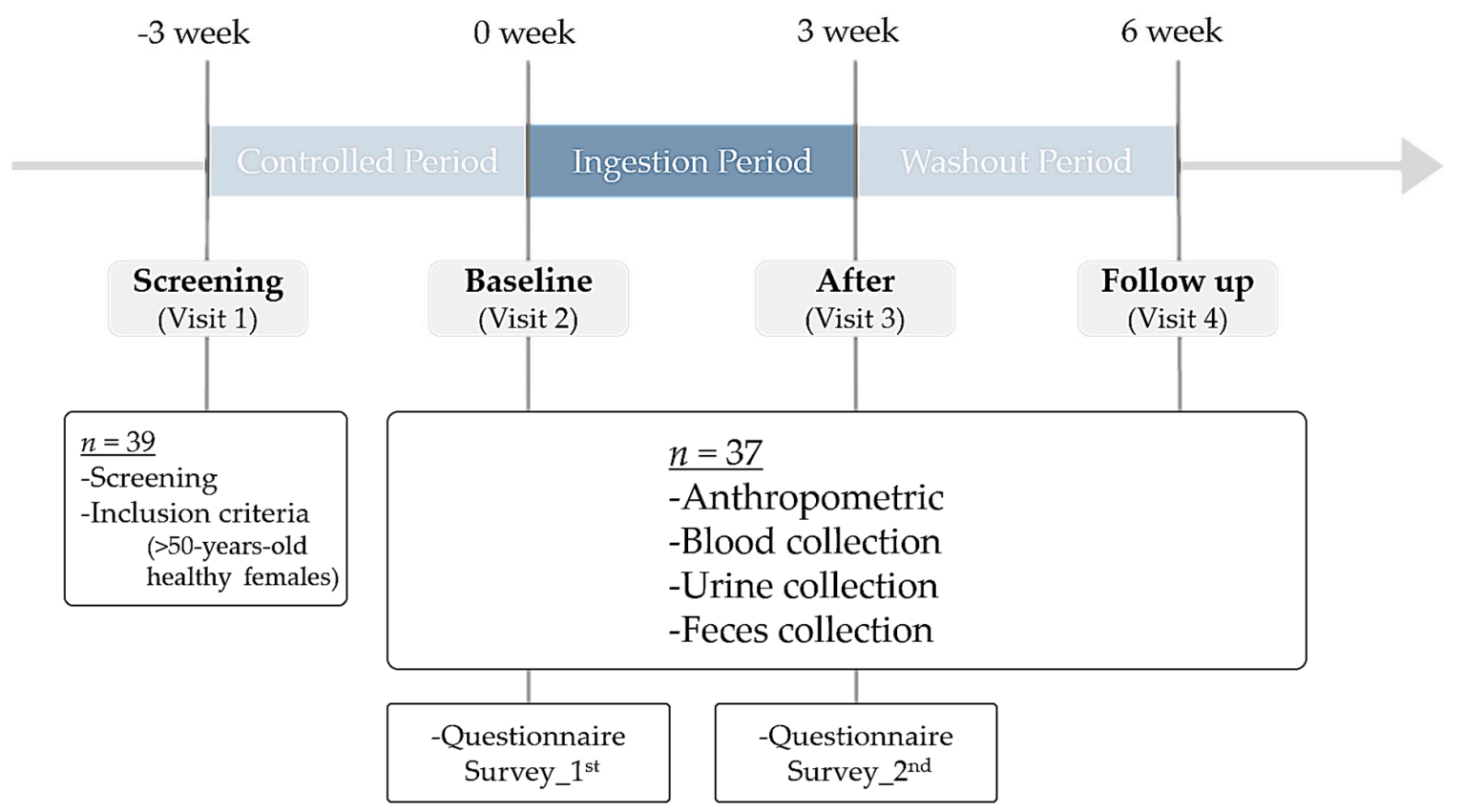

Figure 1. Study flowchart.

\subsection{Contents of the Synbiotic Drink}

The synbiotic drink products (commercial products, MPRO3) used in this study comprised two formulations: a capsule (2ea) and solution $(130 \mathrm{~mL}$ ). The capsule (2ea) contained Bifidobacterium animalis spp. lactis HY8002, Lactobacillus casei HY2782, and L. plantarum HY7712 with crystalline cellulose and lactose. The solution $(130 \mathrm{~mL})$ contained $9800 \mathrm{mg}$ of dietary fiber (polydextrose, chicory dietary fiber, and wheat dietary fiber), $3160 \mathrm{mg}$ of fructo-oligosaccharides, $500 \mathrm{mg}$ of xylo-oligosaccharides, and $350 \mathrm{mg}$ of isomalto-oligosaccharide with the same strains as those in the capsule, along with $1000 \mathrm{mg}$ of lactulose powder and milk crude oil. The total bacterial count was $5.0 \times 10^{9}$ colony-forming units (CFUs) for Bifidobacterium animalis spp. lactis HY8002, $2.5 \times 10^{9}$ CFUs for L. casei HY2782, and $2.5 \times 10^{9}$ CFUs for L. plantarum HY7712. The ratio of bacteria to capsule and solution was 1:2. The products were obtained from Korea Yakult Co. (Gyeonggi-do, Korea). The nutritional composition of the synbiotic drink is stated in Table S2. The quality of all products, including harmful microbial levels, were also checked by Korea Yakult Co.

\subsection{Sample Collection}

Information on food consumption, fasting blood samples, and anthropometric measurements were collected at three intervals: before the study (baseline), after the intake period of 3 weeks (after), and at the end of week 6 (washout).

\subsection{Anthropometric Information}

Weight was measured in the fasting state. Height was measured without shoes to the nearest $0.5 \mathrm{~cm}$, and weight was measured similarly, wearing light clothes, to the nearest $0.1 \mathrm{~kg}$. Waist circumference was measured midway between the lower rib margin and the iliac crest using a non-expandable measuring tape to the nearest $0.5 \mathrm{~cm}$. body mass index (BMI) was calculated as the weight $(\mathrm{kg}$ ) divided by squared height $\left(\mathrm{m}^{2}\right)$ and was interpreted according to the WHO 2000 guideline [3]. A sphygmomanometer was used to measure blood pressure after participants had rested for $5 \mathrm{~min}$ in the sitting position.

\subsection{Blood Sample Collection}

After blood pressure measurement, a blood sample was drawn from the antecubital vein in the arm. Blood samples were collected after overnight fasting and abstinence from any medications, 
tobacco, alcohol, tea, and coffee in EDTA Vacutainer blood collection tubes (Becton Dickinson, Franklin Lakes, NJ, USA) and were mixed. Plasma and red blood cells were separated in the sample via centrifugation $\left(1500 \times \mathrm{g}, 4^{\circ} \mathrm{C}, 15 \mathrm{~min}\right)$. For the measurement of serum biochemical parameters, blood samples collected in EDTA tubes were sent to a hospital (Central Hospital, Seongnam City, Korea) and were immediately analyzed using standard laboratory methods and certified assays.

Biochemical parameters were analyzed in the Central Hospital (Seongnam City, Korea). Glucose (FBG), triglyceride, cholesterol, high-density lipoprotein (HDL), low-density lipoprotein (LDL), LDH, creatinine, alkaline phosphatase (ALP), and C-reactive protein (CRP) were measured using an automated analyzer (Roche Diagnostics, Mannheim, Germany). The remaining blood was stored at $-80^{\circ} \mathrm{C}$. All blood test items including our target blood test items and safety parameters are stated in Table S3.

\subsection{Urine Sample Collection}

Dipstick urinalysis was performed for each urine specimen using a combo stick 10 of a combo stick 720 general urine test strip (DFI, KimJae, Korea). A laboratory technician performed the test within $2 \mathrm{~h}$ of sample collection by placing the dipstick in the urine sample according to the manufacturer's instructions. The results were read within $5 \mathrm{~min}$ [33].

\subsection{Fecal Sample Collection}

We provided the participants with a stool container before each visit. The feces were freshly collected $(0.25 \mathrm{~g})$ the night before or the morning of the visit. The obtained fecal samples were stored in a domestic refrigerator at $4{ }^{\circ} \mathrm{C}$ for less than $4 \mathrm{~h}$ before transportation to the laboratory. The sample was transferred to the laboratory, and DNA was extracted. The samples were then stored at $-80^{\circ} \mathrm{C}$ until analysis.

\subsection{Microbial Analysis}

\subsubsection{DNA Extraction}

DNA extraction was performed using a QIAamp PowerFecal pro-DNA kit (Qiagen, Hilden, Germany) following the manufacturer's recommendations. In brief, a 250-mg aliquot of the fecal sample was transferred to a Dry Bead Tube provided in the kit. Next, $800 \mu \mathrm{L}$ of $\mathrm{C} 1$ solution was added, and the sample was vortexed at the maximum speed for $10 \mathrm{~min}$. The rest of the protocol followed the manufacturer's recommendations. DNA was eluted in a $65-\mu \mathrm{L}$ C6 elution buffer solution. The extracted DNA samples were stored at $-80^{\circ} \mathrm{C}$ until library preparation and sequencing [34].

\subsection{2. $16 \mathrm{~S}$ rRNA Amplicon Library Preparation}

The fecal DNA samples were analyzed by high-throughput 16S rRNA gene amplicon sequencing analysis using an Ion PGM (Thermo Fisher Scientific Inc., Waltham, MA, USA) next-generation sequencer. The V3-V4 regions of $16 \mathrm{~S}$ rRNA genes from each sample were amplified using the following adapter sequences, the index sequence, and general-purpose primers: $341 \mathrm{~F}$ ( $5^{\prime}$-CCT ACG GGN GGC WGC AG-3') with a sample-specific 6-8-base tag sequence and 805R (5'-GAC TAC HVG GGT ATC TAA TCC-3'). Polymerase chain reaction (PCR) was performed with the Platinum PCR SuperMix High Fidelity system (Invitrogen, Carlsbad, CA, USA; Thermo Fisher Scientific Inc., Waltham, MA, USA), using $2.5 \mathrm{ng}$ of template DNA and each primer at a final concentration of $50 \mathrm{nM}$ in a $27 \mu \mathrm{L}$ final reaction volume. PCR was performed under these cycling conditions: $94^{\circ} \mathrm{C}$ for $3 \mathrm{~min}$, followed by 30 cycles of $94{ }^{\circ} \mathrm{C}$ for $30 \mathrm{~s}, 50^{\circ} \mathrm{C}$ for $30 \mathrm{~s}$, and $72{ }^{\circ} \mathrm{C}$ for $30 \mathrm{~s}$. The amplicon libraries were further purified to remove residual primer dimers and any contaminants using the Agencourt AMPure XP DNA purification kit (Beckman Coulter, Brea, CA, USA) following the manufacturer's instructions. Samples were eluted in $15 \mu \mathrm{L}$ low TE buffer. The DNA concentration, quality, and amplicon library concentrations were assessed using the dsDNA high sensitivity assay kit on the Qubit 4 fluorimeter instrument (Invitrogen, 
Carlsbad, CA, USA; Thermo Fisher Scientific Inc., Waltham, MA, USA). The fragment size and quality of the pooled DNA were assessed using an Agilent 2100 Bioanalyzer (Agilent Technologies, Palo Alto, CA, USA). The enriched particles were loaded onto an Ion 554 chip (Thermo Fisher Scientific Inc., Waltham, MA, USA), and sequencing was performed on an Ion PGM according to the manufacturer's instructions [35-38].

\subsubsection{Analysis of $16 \mathrm{~S}$ rRNA Amplicon Sequence}

The FASTAQ file, the raw data of 16S rRNA sequences, was obtained through Ion Torrent Suite Software version 5.14.1.1. (Thermo Fisher Scientific Inc., Waltham, MA, USA) The 16S rRNA workflow module in EzBio cloud software (ChunLab, Inc., Seoul, Korea) 47 was used to classify individual reads by combining the Basic Local Alignment Search Tool with the curated Greengenes database, which contains a high-quality library of full-length $16 \mathrm{~S}$ rRNA sequences. The reads were excluded from the analysis if they were shorter than $500 \mathrm{bp}$ or inappropriately paired. Chimeras were identified using USEARCH software (drive5 bioinformatics software and services, https://www.drive5.com USDOE Office of Science (SC), Biological and Environmental Research (BER) (SC-23), Berkeley, CA, USA) and were removed from the sequence data. Sequences were clustered into operational taxonomic units (OTUs) at 97\% identity using QIIME's pick open reference otus.py and the Greengenes 13.5 reference database and UCLUST algorithm.

\subsection{Statistical Analysis}

Statistical analyses were performed using SPSS (version 20.0; SPSS, Inc., Chicago, IL, USA) and GraphPad Prism, version 8.3.1 for Windows (GraphPad software, San Diego, CA, USA). Normality was verified for each outcome and the corresponding non-parametric tests were conducted on outcomes with a skewed distribution. The changes (mean \pm SD) in individual biochemical indices and microbiota between the baseline and after the intervention and washout period were assessed with a paired $t$-test or a Wilcoxon signed-rank test according to the data distribution. Differences were considered statistically significant at $p<0.05$. Spearman's correlation coefficient (rho, $\rho$ ) was used to determine associations between intra-individual similarities of the microbiota after synbiotic drink intake. PCA (Principal component analysis), a linear dimension reduction method, was used to determine major changes at the genus level, and data were compressed into several informational features that allowed for the two-dimensional visualization of sample similarity. Beta diversities were analyzed using the EzBio cloud dashboard (ChunLab, Inc., Seoul, Korea) [39]. PCA was conducted based on the Jensen-Shannon method [40]. The Ion Reporter suite (Thermo Fisher Scientific Inc., Waltham, MA, USA) was used to filter polymorphic variants. Statistical taxonomic comparisons were performed using linear. discriminant analysis effect size (LEfSe) analysis on the Galaxy Hutlab online platform (Hutlab, Boston, Massachusetts, USA; https://huttenhower.sph.harvard.edu/galaxy) [41,42]. LEfSe is a tool developed to find biomarkers between two or more groups using relative abundances, and linear discriminant analysis (LDA) explicitly attempts to model the difference between the classes of data.

\section{Results}

\subsection{Study Design and Characteristics of Participants}

Characteristics of the 37 participants are summarized in Table 1. All subjects were postmenopausal women aged 65.7 years on average, and all were non-smokers. The study protocol is outlined in Figure 1. All subjects made three official visits during the study period for recruitment and screening. The experimental schedule comprised a baseline, ingestion, and washout phase lasting 9 weeks, with 3-week intervals. In addition, we investigated whether a difference in bacteria aligns with the alcohol consumption pattern in Table 1, but we observed no significant difference. 


\subsection{Blood Parameters and Anthropometric Measurements}

The results of blood parameters and anthropometric measurements are summarized in Table 2. Glucose levels were significantly decreased by $6 \%$ after 3 weeks of synbiotic drink ingestion $(p=0.005)$. Triglyceride (TG), cholesterol, and LDL-cholesterol levels decreased after intake compared to levels at the baseline, but the changes were not statistically significant ( $p=0.474,0.733,0.930$, respectively). Creatinine levels were significantly decreased by $15 \%$ and ALP level was decreased by $3 \%$ after 3 weeks of synbiotic drink ingestion ( $p=0.025,0.047$, respectively). Body weight, BMI, and blood pressure did not differ significantly among measurements at baseline, after the intervention, and during the washout period (Table 2). Biochemical markers of safety were also measured at baseline, after intake, and after the washout period. (Table S4). The results showed comparable levels of all safety biomarkers among the women at the study endpoint, and none showed parameters outside the normal range with clinically significant values.

Table 2. Changes of anthropometric measurements, biochemical parameters, and fecal characteristics at each timepoint ${ }^{1}$.

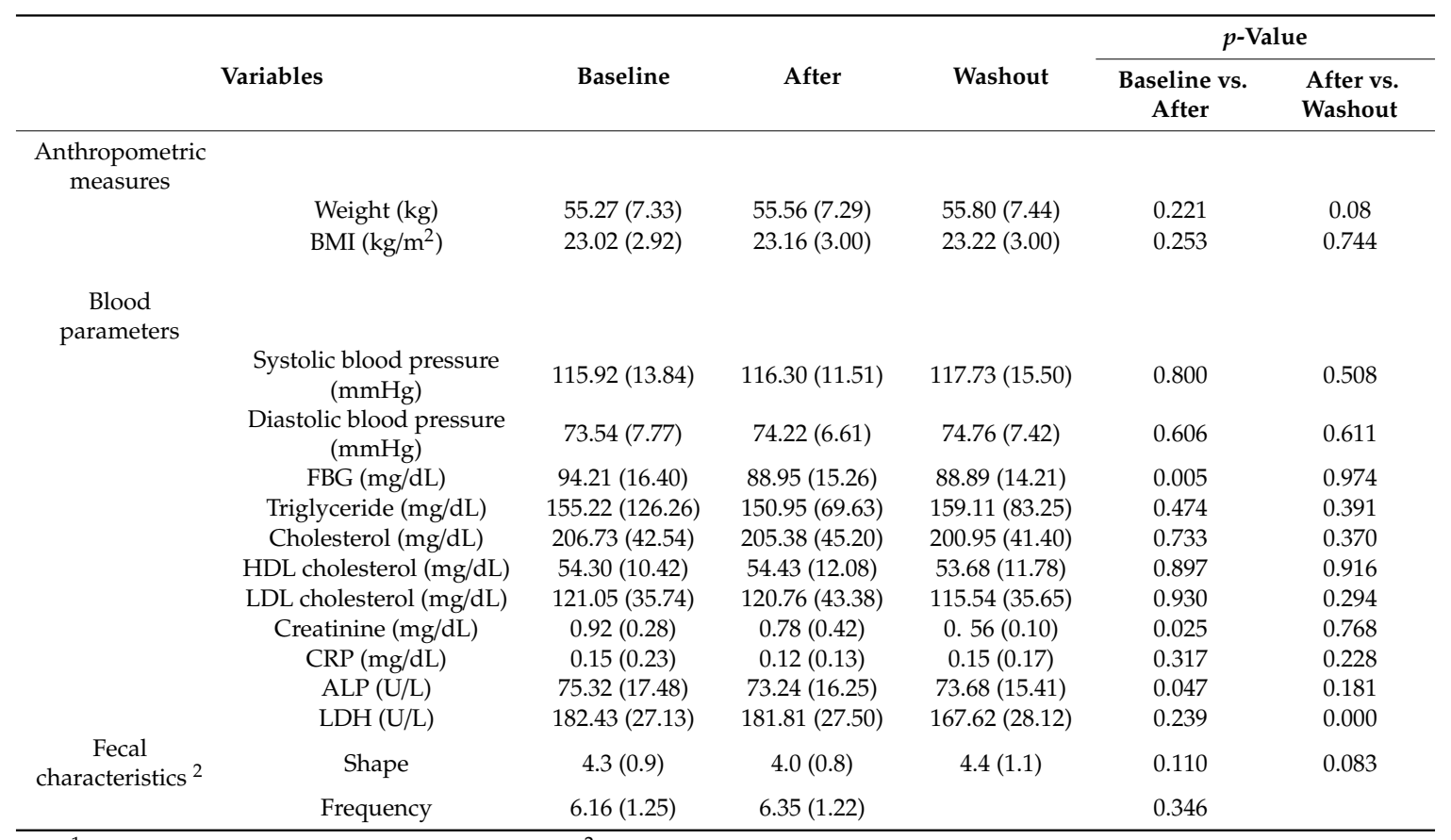

${ }^{1}$ All values are presented as mean $( \pm \mathrm{SD}) .{ }^{2}$ Fecal characteristics are based on the standard Bristol stool level. Abbreviations: BMI, body mass index; FBG, fasting blood glucose; CRP, C-reactive protein; HDL, high-density lipoprotein; LDL, low-density lipoprotein; ALP, alkaline phosphatase; LDH, lactate dehydrogenase.

\subsection{Fecal Assessment}

We investigated the mean level of fecal characteristics for 3 weeks before and after the intervention, as well as after the washout period (Figure S1). The average defecation frequency was calculated by the number of defecations per week. Compared with that at baseline, the proportion of subjects with a daily defecation frequency increased from $62 \%$ to $70 \%$ after the intervention. The average defecation frequency increased from 6.16 to 6.35 times per week. Further, the proportion of women with a BSFS 4 (sausage) form of feces increased from $43 \%$ to $68 \%$ and then returned to $30 \%$ at the washout timepoint (Figure S1) $[43,44]$. The raw data of the fecal type of the subjects are shown in Table S5.

\subsection{Intestinal Microbial Community Analysis}

Based on the OTUs, the relative abundances of bacteria at the phylum and genus levels were visualized as a pie chart and bar graph, respectively, as shown in Figure 2. The OTU raw data are 
provided in Table S6. After the intervention, the proportions of Firmicutes and Proteobacteria were lower than those detected at pre-intervention ( $81.3 \%$ vs. $85.5 \%$ and $1.1 \%$ vs. $1.2 \%$, respectively), albeit without statistical significance. However, the proportions of Actinobacteria and Bacteroidetes were significantly higher than those measured at baseline $(9.0 \%$ vs. $7.8 \%$ and $8.5 \%$ vs. $5.3 \%$, respectively; $p=0.026)$. Changes in the gut microflora after ingestion reverted to the baseline again during the washout period (Figure 2A).

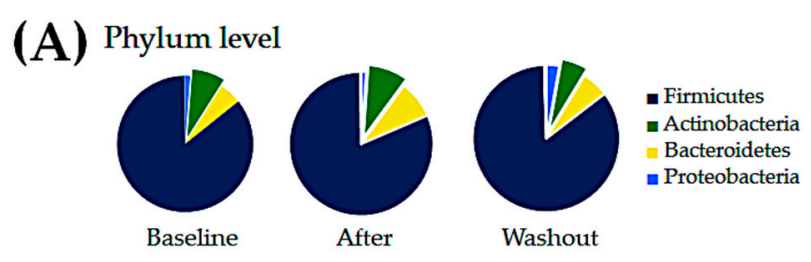

(B)

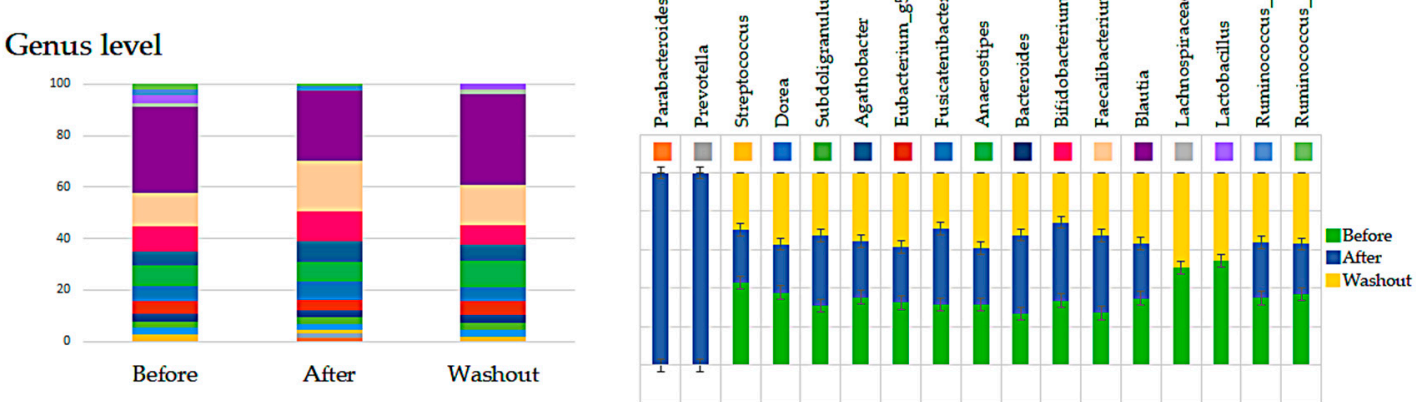

(C) Specific species level
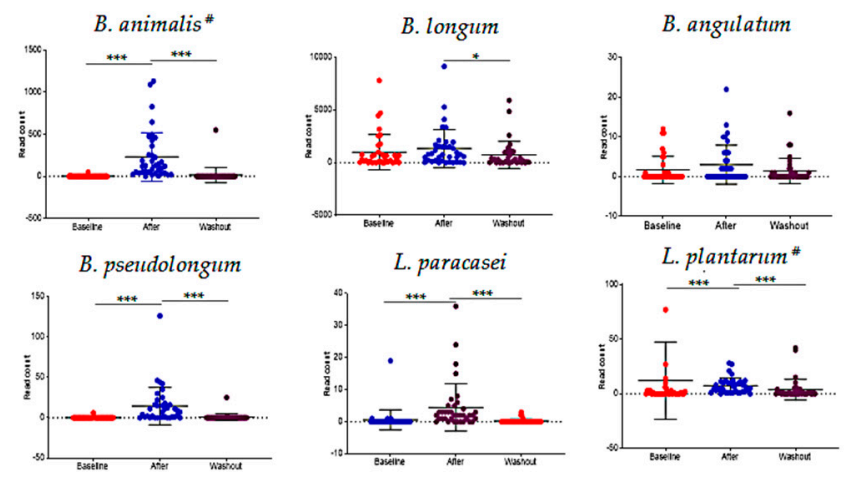

Figure 2. Relative abundance of intestinal microbial phyla (A), genera (B), and species (C) in 37 participants based on $16 \mathrm{~S}$ rRNA analysis. Numbers indicate the relative contribution (percentage) of each microbial level. ${ }^{*} p<0.05,{ }^{* * *} p<0.005$ (paired $t$-test and Wilcoxon rank-sum test). In (C), Crosshatch $(\#)$ is indicated for the bacteria contained in the synbiotic drink.

At the genus level, the relative abundance of Bifidobacterium was significantly increased after the intervention (8.4\%) compared to that at baseline (5.3\%) and then decreased again after washout $(6.9 \%$, $p=0.017)$. Faecalibacterium and Fusicatenibacter increased after the intervention $(13.8 \%, p=0.009 ; 5.1 \%$, $p=0.039)$ compared to the baseline values (11.1\% and $3.7 \%$, respectively), and decreased again after washout $(9.2 \%$ and $4.1 \%$, respectively; $p=0.014)$. In contrast, Blautia and Eubacterium_g5 decreased after the intervention (19.3\% and $2.9 \%$, respectively) compared to the baseline levels $(25.1 \%$ and $3.9 \%)$ and significantly increased again after washout $(23.7 \%, p=0.005 ; 3.3 \%, p=0.008)$. No significant changes were observed in the relative abundance of other genera, with changes only observed after the intervention (Figure 2B). 
We also analyzed the most significant species of Bifidobacterium and Lactobacillus, both of which are in the synbiotic drink formulation. B. animalis and L. plantarum, which are in the synbiotic drink, increased significantly after ingestion $\left(p=4.20 \times 10^{-13}\right.$ and $p=2.40 \times 10^{-4}$, respectively) and decreased again during the washout period $\left(p=2.00 \times 10^{-12}\right.$ and $p=2.30 \times 10^{-5}$, respectively). Bifidobacterium pseudolongum increased significantly after ingestion $\left(p=2.50 \times 10^{-8}\right)$ and decreased again during the washout period $\left(p=6.50 \times 10^{-8}\right)$. L. paracasei increased significantly after ingestion $\left(p=1.80 \times 10^{-7}\right)$ and then decreased again during the washout period $\left(p=2.00 \times 10^{-7}\right)$. However, the relative abundance of Bifidobacterium longum did not change after ingestion and significantly decreased again during the washout period ( $p=0.035$; Figure 2C). P-values for all items in Figure 2 are stated in Table S7.

\subsection{Temporal Changes in the Intestinal Microbiota}

As a complementary and validation method to identify differentially expressed taxa, we applied LDA LEfSe analysis, which generated an LDA score and cladogram for visual representation of the results (Figures 3-5).

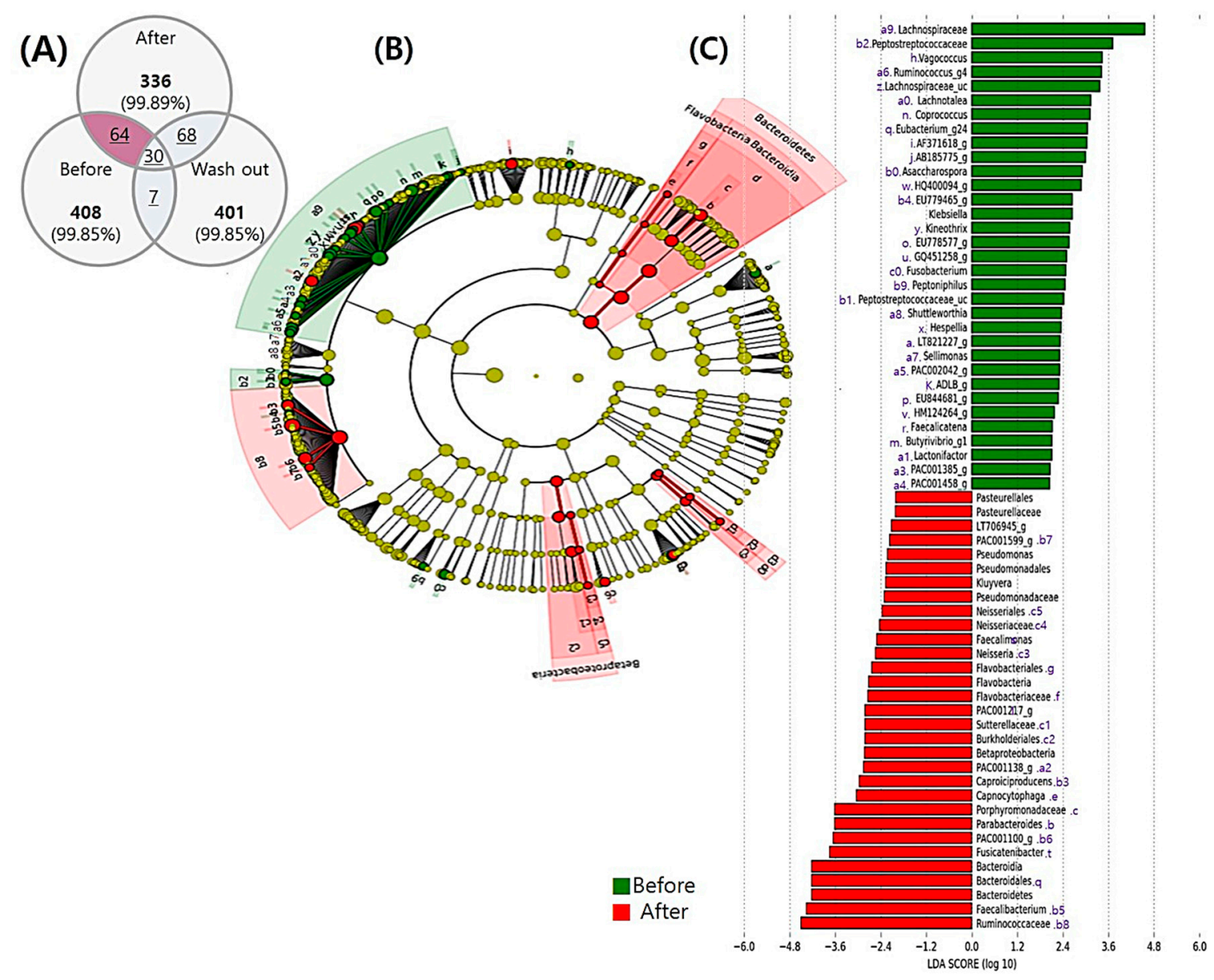

Figure 3. Cross-taxa profile of intestinal microflora in all subjects $(n=37)$ before and after synbiotic ingestion. (A) Venn diagram for the distribution of operational taxonomic units (OTUs) at each time point. (B) Cladogram showing phylogenetic relationships among taxa with statistically significant differences between time points. (C) Linear discriminant analysis effect size (LEfSe) showing the contribution of different bacteria to the differences in the before and after period. Green color indicates taxa enriched before ingestion, and red color indicates taxa enriched after synbiotic ingestion. 


\subsubsection{Comparison before and after the Intervention}

We found 64 OTUs based on the LDA scores for the effect size of taxonomic groups with relative abundance ratios before and after synbiotic intake and generated bar graphs (Figure 3). Regarding the intestinal microbial abundance before synbiotic drink intake (baseline), at the family level, Lachnospiraceae and Peptostreptococcaceae showed the highest diversity. At the genus level, we identified 24 genera of Lachnospiraceae. We also identified two prevailing genera of Peptostreptococcaceae, namely Asaccharospora and Peptostreptococcaceae_uc. Moreover, Vagococcus was the most dominant genera that did not belong to the abovementioned families. Klebsiella, Fusobacterium, and Peptoniphilus were also observed (Figure 3B,C).

After ingestion of the synbiotic drink, Bacteroidetes was observed at the phylum level. At the class level, Bacteroidia of Betaproteobacteria and Bacteroidetes showed the highest diversity. At the order level, Pasteurellales, Pseudomonadales, Neisseriales, Flavobacteriales, and Burkholderiales were dominant. At the family level, Pasteurellaceae, Pseudomonadaceae, Neisseriaceae, Flavobacteriaceae, Sutterellaceae, Porphyromonadaceae, Bacteroidales, and Ruminococcaceae were dominant. At the genus level, Pseudomonas, Kluyvera, Faecalimonas, Neisseria, Flavobacteria, Caproiciproducens, Capnocytophaga, Parabacteroides, Fusicatenibacter, and Faecalibacterium were predominant. Further, we observed higher abundance at various levels of relative diversity after synbiotic drink intake (Figure 3).

\subsubsection{Comparison between the end of Intervention and Washout Period}

Sixty-eight OTUs were found and a bar graph was generated based on the LDA scores for the effect size of the taxonomic group with relative abundance at washout and after synbiotic drink intake (Figure 4). After ingestion of the synbiotic drink, there was a prevalence of Actinobacteria at the phylum level, Actinobacteria_c at the class level, Bifidobacteriales at the order level, Bifidobacteriaceae at the family level, and Bifidobacterium at the genus level. In addition, Epsilonproteobacteria was prevalent at the class level, Campylobacterales at the order level, Campylobacteraceae at the family level, and Campylobacter at the genus level. At the genus level, Sellimonas and Aggregatibacter were also prevalent.

At washout after ingesting the synbiotic drink, at the family level, Lachnospiraceae and its genera Blautia, Eubacterium, Faecalimonas, Eubacterium, and Howardella were predominant. Terrisporobacter and Porphyromonas genera were also dominant. Finally, at the phylum level, Saccharibacteria_TM7 was dominant, with Saccharimonas_c at the class level, Saccharimonas_o at the order level, and Saccharimonas at the family level (Figure 4B,C). We additionally compared the washout period with the baseline period before synbiotic drink consumption. However, most profiles matched between these two phases and no significantly different bacteria were found in this analysis (Figure S2).

\subsection{Comprehensive Comparison of Each Time Point and Associations among All Parameters and Microbiota Composition}

The intestinal microbial community changes differed in the comparison between baseline and after ingestion and between after ingestion and washout. We found 30 bacteria that changed only after intake, 20 bacteria that showed increased abundance, and 10 that showed decreased abundance (Figure 5B).

At the family level, the increased bacteria predominantly included seven Lachnospiraceae, six Ruminococcaceae isolates, and Sutterellaceae. At the genus level, Faecalibacterium, Fusicatenibacter, Faecalimonas, and Caproiciproducens were predominant. Lachnospiraceae was the most dominant family of bacteria, the abundance of which decreased after ingestion. At the genus level, the bacteria that decreased after ingestion at high rates were Blautia, Eubacterium_g24, and Eubacterium_g5. We further assessed the relationship between 16 genera and all parameters, including blood values, which were changed by the intervention (Figure 5C). We plotted a heat map using the Spearman correlation coefficient to evaluate how these variables are related. The relative abundance of the 
genus Ellagibacter of the family Eggerthellaceae was negatively correlated with BMI (rs $=-0.331$, $p=0.045)$. Terrisporobacter of the family Peptostreptococcaceae was positively correlated with CRP ( $\mathrm{rs}=0.353, p=0.032), \mathrm{TG}(\mathrm{rs}=0.365, p=0.026), \mathrm{BMI}(\mathrm{rs}=0.493, p=0.002)$, and body weight $(\mathrm{rs}=0.425$, $p=0.009$ ) and was negatively correlated with high-density lipoprotein cholesterol (HDL-C) (rs $=-0.380$, $p=0.020)$. Acutalibacter of the family Ruminococcaceae was positively correlated with fecal shape ( $\mathrm{rs}=0.566, p=0.000)$. Caproiciproducens of the family Ruminococcaceae and Eubacterium_g5 of the family Lachnospiraceae showed negative correlations with fecal frequency $(\mathrm{rs}=-0.405, p=0.013$ and rs $=-0.339, p=0.040$, respectively). Lachnospiraceae_uc, Eubacterium_g5, and Blautia of the family Lachnospiraceae were positively correlated with creatinine ( $\mathrm{rs}=0.352, p=0.033 ; \mathrm{rs}=0.338$, $p=0.041$; and rs $=0.456, p=0.005$, respectively), and PAC001100_g of the family Ruminococcaceae was negatively correlated with creatinine ( $\mathrm{rs}=-0.415, p=0.011$ ). The changes in fecal shape and frequency were positively correlated with changes in Caproiciproducens and Eubacterium_g5 abundance, but were negatively correlated with changes in Acutalibacter. The changes in BMI, weight, and TG were negatively correlated with changes in Terrisporobacter. Further, significantly positive correlations were observed between creatine and changes in Lachnospiraceae_uc ( $\mathrm{rs}=0.352, p=0.033)$, Eubacterium_g5 ( $\mathrm{rs}=0.388, p=0.041$ ), and Blautia ( $\mathrm{rs}=0.456, p=0.005)$, and a negative correlation was found between this parameter and changes in PAC001100_g ( $\mathrm{rs}=-0.415, p=0.011)$.

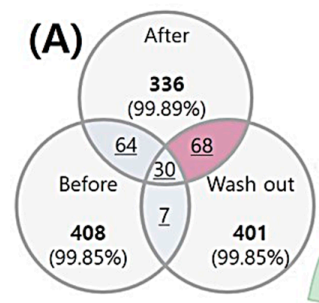

(B)
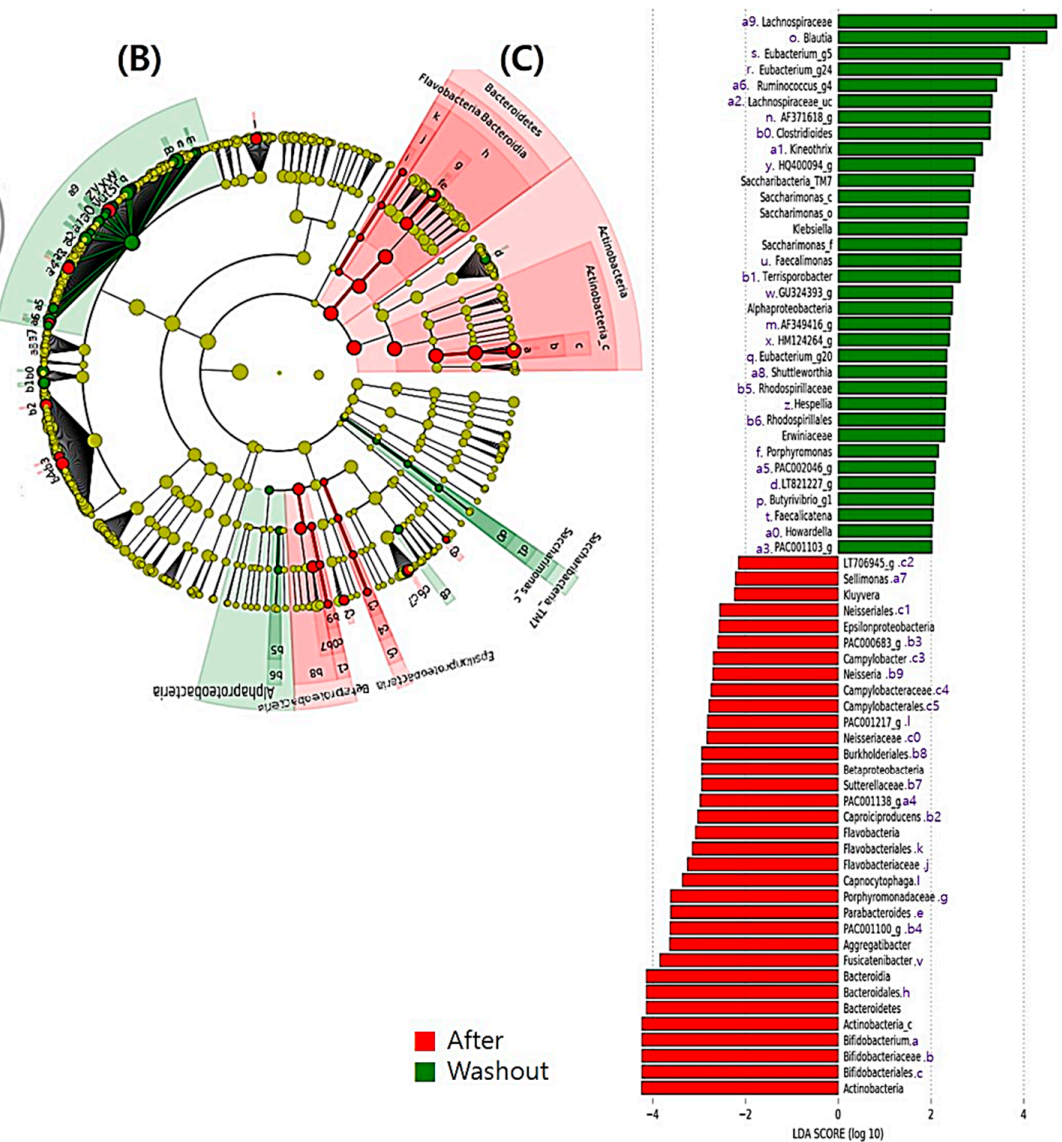

Figure 4. Cross-taxa profile of intestinal microflora in all subjects $(n=37)$ after synbiotic ingestion and during washout. (A) Venn diagram for the distribution of operational taxonomic units (OTUs) at each time point. (B) Cladogram showing phylogenetic relationships between taxa with significant differences between time points. (C) Linear discriminant analysis effect size (LEfSe) showing the contribution of different bacteria to the differences between the after ingestion and washout periods. Green color indicates taxa enriched after ingestion, and red color indicates taxa enriched in the washout period. 


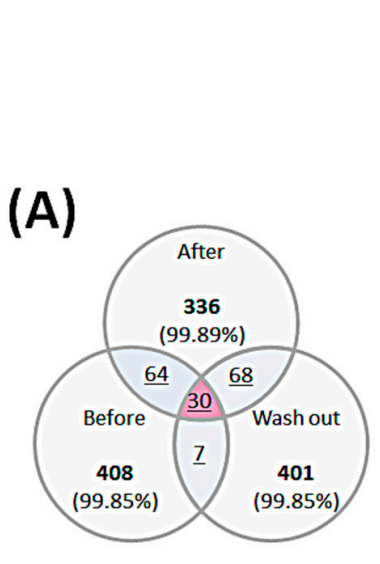

(B)
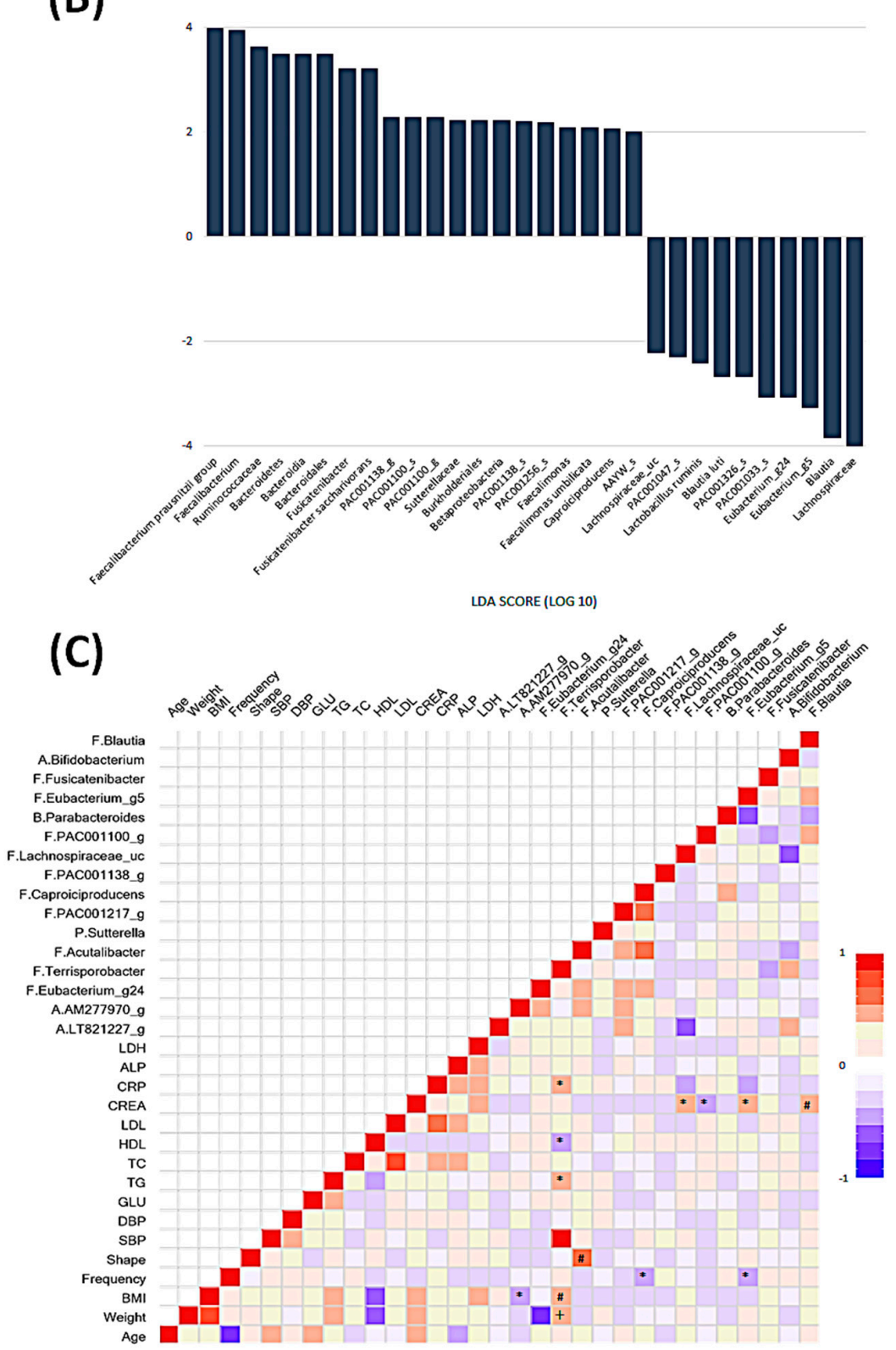

Figure 5. Cross-taxa profile of intestinal microflora and biological parameters in all subjects $(n=37)$ compared among the three periods (before, after, and washout). (A) Venn diagram showing the distribution of operational taxonomic units (OTUs) for each time point. (B) Based on the linear discriminant analysis (LDA) score, 30 significantly altered bacteria represent an increased and decreased group, including 16 genera with significance and tendencies toward significance. (C) Heat map of the Spearman rank correlations between biological and gut microbial outcomes. Red indicates a positive correlation and blue indicates a negative correlation. The heat map shows the first letter of the phylum-level bacteria and genus-level bacteria in order. A, Actinobacteria; F, Firmicutes; P, Proteobacteria; B, Bacteroidetes. ${ }^{*} p<0.05, \# p<0.01$, and $+p<0.0005$ (after false discovery rate correction). Abbreviations: BMI, body mass index; SBP, systolic blood pressure; DBP, diastolic blood pressure; GLU, glucose; TG, triglyceride; TC, total cholesterol; HDL, high-density lipoprotein; LDL, low-density lipoprotein; CREA, creatinine; CRP, C-reactive protein; ALP, alkaline phosphatase; LDH, lactate dehydrogenase. 


\section{Discussion}

Countries with high life expectancy are now fighting multiple causes of aging by focusing on diet, hoping to extend human life to 100 years or over. Developing preventive nutritional strategies to maintain or improve the quality of life of an aging population is essential and timely [45-48]. Several groups are studying diet control in the elderly, and recent reports have focused on the positive relationship between human health and the microbial flora of the human gut [49-52].

Among the gut bacteria that act as functional organisms in humans, probiotics are worth studying because they have a wide range of beneficial effects on host health and longevity. Bifidobacteria, which have been studied by many researchers, decrease as aging progresses and have been an indicator of effectiveness when ingesting probiotics [53-55]. However, in our study, the ingestion of a synbiotic drink did not increase the abundance of these bacteria in all subjects. Nevertheless, in those who did not experience an increase, physiological effects beneficial to health, including fecal activity, were observed. Therefore, our results agree with the reports that the mechanisms by which probiotics influence host longevity are not yet clear.

We emphasized the minimization of variables, which is the limitation mentioned by many studies on probiotics. Changes from phylum to genus level were observed through intestinal microbial community analysis, and in this study, relatively different microbial community ratios were observed. To observe the tendency of microbial changes, we further analyzed the intestinal microbes at the time of washout. The results showed changes in certain communities only after ingestion, which then returned to the baseline levels at washout.

We found 30 bacteria in this study that only change when consumed as a synbiotic drink. These 30 bacteria, which change at the after timepoint, are noted only after synbiotic drink consumption. Among them, six genera and several blood index values were observed to have a consistent tendency. Ellagibacter abundance in all participants was associated with BMI. Terrisporobacter was associated with CRP, TG, BMI, and HDL-C. Lachnospiraceae_uc, Eubacterium_g5, Blautia, and PAC001100_g were associated with creatinine.

The genus Ellagibacter of the Eggerthellaceae family did not appear prior to consumption of the synbiotic drink, but detected after consumption of the synbiotic drink. It did not appear again in washout. Urolitin is known as an intestinal microbial metabolite produced from ellagic tannins and foods containing ellagic acid such as walnuts, strawberries, and pomegranates [56,57]. The health benefits associated with these metabolites vary considerably from individual to individual, depending on the composition of the gut microbiota. Urolitin has several properties, including anti-inflammatory, neuroprotective, cardioprotective, "prebiotic-like", anti-diabetes, anti-obesity, antioxidant and chemopreventive activity, and improved muscle function through various in vitro and animal studies [56]. To date, two genera of bacteria of the human intestine belonging to the family Coriobacteriaceae have been identified as urolithin producers. It is an interesting finding in this study that one of the two bacterial genera, Ellagibacter, appears only when ingested in synbiotic drinks. Ellagibacter, found at the time point after ingestion of the synbiotic drink, had a negative correlation with BMI, and those with high Ellagibacter had lower BMI levels at the time point after ingestion of the synbiotic drink. However, even if there is a correlation between Ellagibacter and BMI, no improvement in BMI was observed in our study. In addition, so far, little has been investigated whether Ellagibacter correlates with BMI improvement in vivo [58]. In another study, urolithin was divided into three phenotypes, and found that it appeared differently depending on the health status of participants and demographic characteristics such as age, sex, and BMI. These urolithin phenotypes may differ in the human gut microbiota, and whether they should be considered in clinical trials and could be biomarkers associated with other health benefits or disease predisposition, revealed that further research is needed [59]. Therefore, the intake of a synbiotic drink for a short period of time causes Ellagibacter to appear, and whether the intake of a synbiotic drink improves BMI is not known in this study, so a follow-up study is needed. 
The Peptostreptococcaceae family Terrisporobacter decreased after consumption of a synbiotic drink and increased again after stopping consumption. This finding is similar to the findings of studies involving prebiotic administration in neonatal pigs. When comparing the prebiotic intervention group and the control group, Terrisporobacter was more prevalent in the control group after prebiotic administration [60]. The mechanism by which consumption of synbiotic beverages decreases Terrisporobacter abundance should be studied. At the time point after ingestion of a synbiotic drink, Terrisporobacter showed positive correlation with CRP, TG, BMI, and body weight, and negative correlation with HDL-C. Participants with low Terrisporobacter abundance had low CRP, TG, BMI, and weight levels. Conversely, HDL-C levels were high. However, although there was a correlation between Terrisporobacter and BMI and HDL-C, there was no improvement in this study. Due to the lack of research on Terrisporobacter, our results suggest that future studies on Terrisporobacter are needed to explore the underlying mechanisms involved in these effects on anthropometric measurements.

Lachnospiraceae_uc, Eubacterium_g5, and Blautia decreased only after ingestion of a synbiotic drink, and increased again after stopping intake. PAC001100_g of the Ruminococcaceae family increased only after intake of a synbiotic drink, and decreased again after stopping intake. Other studies have shown that clustering of Lachnospiraceae induced a significant increase in hepatic and mesenteric adipose tissue weight, as well as rat fasting blood glucose levels, and decreased plasma insulin levels and HOMA- $\beta$ values. It indicates that Lachnospiraceae has an effect on the development of obesity and diabetes [61]. In addition, Lachnospiraceae and Blautia were abundant in NAFLD cirrhosis patients [62]. There are not many studies on Eubacterium. Lachnospiraceae_uc, Eubacterium_g5, Blautia and creatinine showed positive correlations. Participants with low levels of Lachnospiraceae_uc, Eubacterium_g5, and Blautia also had low creatinine content. PAC001100_g negatively correlated with creatinine, and people with high PAC001100_g levels had low creatinine levels. Although this correlation was found, it was only significant in baseline and after timepoint comparisons. A study that analyzed the composition and function of gut microbiota in fecal samples from bowel transplant recipients compared to 84 patients with chronic kidney disease (CKD) and 53 healthy subjects, showed that it was negatively correlated with clinical markers such as several beneficial genus serums of Lachnospiraceae. It has been shown to be creatinine [63]. Other studies have also shown that intestinal dysbiosis occurs in CKD and can actively contribute to the progression of renal failure [64]. The main characteristic of CKD dysbiosis is an increase in Proteobacteria [65], but an increase in Lachnospiraceae has also been observed [66,67]. Specifically, we identified four strains that affect serum creatinine, which were only altered by consumption of probiotics such as synbiotics or synbiotic drinks. Therefore, these strains changed after ingestion of synthetic beverages for 3 weeks, which was closely related to creatinine regulation.

Overall, significant changes in the intestinal microbial community were only observed after ingestion. When comparing the gut microbiota between baseline and washout timepoint, little difference was found. This is explained by people having a unique microbial community that is always maintained if there is no change in diet and dietary habits. Thus, supplements containing synbiotics can change the gut microbiota even with short-term intake. Some of the blood indicators (FBG, creatinine, ALP) were observed to be improved even during the washout period after the consumption of the synbiotic drink. The results of one study showed an increase in glucose metabolism for several months beyond the period of probiotic consumption. This allows microbes to process dietary polysaccharides that are not digested by human enzymes, which can affect the metabolism of additional glucose into the pool of glucose that can be absorbed by the stomach. In addition, probiotics elicit powerful anti-inflammatory capabilities by inhibiting the NF-kB pathway, which mediates microbial activation of the immune system through toll-like receptors [68]. In studies on creatinine, a decrease in creatinine may be affected by loss of appetite [69], and an increase in creatinine may be due to intake of angiotensin-converting enzyme inhibitors (ACE inhibitors) [70,71]. Other clinical studies have also shown results that extend the effect to several months beyond the period of probiotic consumption. In that study, the effects of probiotic interventions lasted up to 7 years in reducing the risk of atopic 
eczema [72]. There are currently few reports on the effects of probiotics on blood ALP. ALP is generally higher in patients with liver disease but decreased in this study. Although not a clinical study, one study noted that mineral deficiency in fish was correlated with low alkaline phosphatase activity in plasma or serum [73]. Other studies have shown that lactic acid bacteria and Bifidobacteria vary from country to country and dietary effects are also important. In a study examining the nutritional evaluation of Koreans, most Koreans eat kimchi, vegetables, pastes, and pickles every day [74]. A characteristic feature of the Korean diet is that it consumes a lot of fermented foods, including kimchi, a traditional Korean fermented vegetable dish [75]. It has potential probiotic effects such as cholesterol reduction, antioxidant properties, and antimicrobial activity [76]. Most of the participants in our questionnaire were on a vegetable-based diet, including kimchi, and participants were instructed to maintain their usual diet for the entire study period. Additionally, all participants were elderly and sensitive to general dietary management. Therefore, the maintenance of some of the improved blood indices even during the vacation period is explained by the possibility that the Korean-specific diet or efforts to improve the diet against aging of the elderly were involved.

Overall, we found changes in clinical parameters associated with many changes in total microbial function because of synbiotic drink intake for only 3 weeks. Nevertheless, aspects of the study design should be considered when interpreting these results. This study was a longitudinal panel study with a design suitable for observing changes over time in the same population. Moreover, we tried to avoid many unexpected variables. To maintain food freshness and high adherence to the intervention, the food was distributed once per week and continuous monitoring by research personnel was conducted. Along with targeted blood tests, the safety of subjects was assessed based on blood safety parameters. Although the questionnaire was not administered at every visit, since the content of the questionnaire administered at the two visits was different, it was an index to evaluate the participants' interest in the research and participation compliance.

The primary strength of this study was that it was not a cross-sectional study but a panel study with a longitudinal design, monitoring the same individuals with the same measurement tools through repeated measurements at different time points, which facilitated data analysis before and after the study. Most probiotic studies compare only before and after consumption. Moreover, most studies suggest that it is difficult to determine whether the altered factors are because of ingested probiotics or other variables, such as changes in dietary habits, antibiotic use, and factors in the external environment. In this study, numerous bacteria changed after ingestion compared to levels before the ingestion period. However, during the washout period, we focused on finding bacteria whose abundance returned to levels observed before ingestion (i.e., the bacteria that changed only after ingesting the synbiotic drink). This is because strains not returning to these levels, are not associated with the synbiotic drink effects.

This study cohort comprised elderly women with a higher intake and interest in probiotics; hence, their compliance to the specific dietary conditions was high. Owing to differences in the behavioral radius, lectures were conducted at the senior complex for several days each week, thus helping researchers better understand various factors in this population, such as the frequent use of drugs, supplements, or antibiotics that could interfere with the gut microflora. None of the participants died or dropped out during the 9-week study period. Overall, various measurements and items were measured in the anthropometric and blood index areas, and emphasis was placed on observing the intestinal microbes that changed only after ingesting the synbiotic drink. Future studies on metagenomic sequencing are thus warranted to target and uncover the associations observed.

The major limitations of this study are the relatively small number of participants and the lack of separate control groups. However, because this was a panel study, the sample population remained constant throughout the study. This was therefore considered the baseline control before the start of the study, thus forming a robust control group. 


\section{Conclusions}

Short-term ( 3 weeks) intake of a synbiotic drink improved participants' satisfaction with their stool shape, bowel activity, and bowel health improvement. We observed that even short-term consumption of a synbiotic drink can alter the intestinal microbial composition of elderly Korean women. In addition, by stopping and observing the intake, we found 30 significant microbes that only changed intake of the synbiotic drink. Continuous consumption of synbiotics is an important prerequisite for this effect. Synbiotics can be a preventive food supplement for chronic diseases that affect healthy older people. Further research is needed to target specific microbial and blood indicators to investigate the combined effects of synbiotics, probiotics, and prebiotics, along with the causal and clinical effects that are responsible for these changes.

Supplementary Materials: The following are available online at http://www.mdpi.com/2072-6643/12/10/3112/s1, Figure S1: Changes of fecal characteristics during the intervention. Figure S2: Cross-taxa profile of intestinal microflora before ingestion of synbiotic drink and at wash out time point in all subjects $(n=37)$. (A) Venn diagram for the distribution of operational taxonomic units (OTUs) at each time point. (B) Cladogram showing phylogenetic relationships among taxa with statistically significant differences between time points. (C) Linear discriminant analysis effect size (LEfSe) showing the contribution of different bacteria to the differences in the before and after period. Green color indicates taxa enriched before ingestion, and red color indicates taxa enriched after synbiotic ingestion. Table S1: Questionnaire raw data, Table S2: The ingredient list of synbiotic drink, Table S3: All subjects' body measurements, blood, and urine test results data, Table S4: Comparison of biochemical markers of safety (mean \pm SD) at baseline, after and at the endpoint of the trial, Table S5: Data for observing the change in stool shape at each time point in all subjects, Table S6: OTUs raw data, Table S7: Relative abundance and $p$ value of intestinal microbial phyla (A), genera (B), and species (C) in 37 participants based on 16S rRNA.

Author Contributions: S.H.L. and H.S.Y. conducted the research, analyzed the data, and wrote the manuscript; H.-G.K. and S.S.K. designed the research; S.H.H. contributed to review of manuscript. All authors have read and agreed to the published version of the manuscript.

Funding: We received no gifts of materials or any additional support.

Acknowledgments: We thank the participants who participated in and provided fecal samples for the study. We also thank the staff at the Miraeseum Seongnam senior complex for their cooperation in this study. We appreciate the technical assistance for operating blood test equipment from Seongnam Central Hospital. We thank the Korea Yakult Co. for generously supplying the synbiotics for our trial and the study participants. This research was supported by the Bio \& Medical Technology Development Program of the NRF funded by the Korean government, MSIP (Grant No. 2016M3A9B694241). This article was revised by a professional English proofreader (Editage).

Conflicts of Interest: The authors declare no conflict of interest.

\section{References}

1. Korea National Statistical Office. Future Population Estimation. Future Life Expectancy/Nationwide. Korea: Population Trend Division, Statistics Korea. Available online: http://kosis.kr/statHtml/statHtml.do?orgId= 101\&tblId=DT_1BPA201\&vw_cd=MT_ZTITLE\&list_id=A41_10\&seqNo=\&lang_mode=ko\&language= kor\&obj_var_id=\&itm_id=\&conn_path=MT_ZTITLE (accessed on 28 March 2019).

2. Korea National Statistical Office. Life Table, National Approval Statistics No. $101035 . \quad$ Expected Life Expectancy (0-Year-Old Life Expectancy) and Life Expectancy (Excluding Health Period). Korea: Population Trend Division, Statistics Korea. Available online: http://www.index.go.kr/potal/stts/idxMain/ selectPoSttsIdxSearch.do?idx_cd=2758\&stts_cd=275801\&freq $=Y$ (accessed on 5 December 2017).

3. Wang, K.; Yu, X.H.; Li, Y.; Guo, Y.; Ge, L.; Pu, F.F.; Ma, X.Y.; Cui, W.J.; Marrota, F.; He, F.; et al. Bifidobacterium bifidum TMC3115 Can Characteristically Influence Glucose and Lipid Profile and Intestinal Microbiota in the Middle-Aged and Elderly. Probiotics Antimicrob. Proteins 2019, 11, 1182-1194. [CrossRef] [PubMed]

4. Ohkusa, T.; Koido, S.; Nishikawa, Y.; Sato, N. Gut Microbiota and Chronic Constipation: A Review and Update. Front. Med. 2019, 6, 19. [CrossRef] [PubMed]

5. Saito, I. Epidemiological Evidence of Type 2 Diabetes Mellitus, Metabolic Syndrome, and Cardiovascular Disease in Japan. Circ. J. 2012, 76, 1066-1073. [CrossRef] [PubMed]

6. Panigrahi, P.; Parida, S.; Nanda, N.C.; Satpathy, R.; Pradhan, L.; Chandel, D.S.; Baccaglini, L.; Mohapatra, A.; Mohapatra, S.S.; Misra, P.R.; et al. A randomized synbiotic trial to prevent sepsis among infants in rural India. Nature 2017, 548, 407-412. [CrossRef] [PubMed] 
7. Anderson, A.D.G.; McNaught, C.E.; Jain, P.K.; MacFie, J. Randomised clinical trial of synbiotic therapy in elective surgical patients. Gut 2004, 53, 241-245. [CrossRef] [PubMed]

8. Abney, K.N.; Susan, J.H. Probiotic supplementation and reducing inflammation in hemodialysis patients: A systematic review. J. Ren. Nutr. Metab. 2019, 5, 3. [CrossRef]

9. Dore, M.P.; Bibbo, S.; Fresi, G.; Bassotti, G.; Pes, G.M. Side Effects Associated with Probiotic Use in Adult Patients with Inflammatory Bowel Disease: A Systematic Review and Meta-Analysis of Randomized Controlled Trials. Nutrients 2019, 11, 2913. [CrossRef] [PubMed]

10. Hou, Q.C.; Zhao, F.Y.; Liu, W.J.; Lv, R.R.; Khine, W.W.T.; Han, J.; Sun, Z.H.; Lee, Y.K.; Zhang, H.P. Probiotic-directed modulation of gut microbiota is basal microbiome dependent. Gut Microbes 2020, 12, 1736974. [CrossRef]

11. Kristensen, N.B.; Bryrup, T.; Allin, K.H.; Nielsen, T.; Hansen, T.H.; Pedersen, O. Alterations in fecal microbiota composition by probiotic supplementation in healthy adults: A systematic review of randomized controlled trials. Genome Med. 2016, 8, 52. [CrossRef]

12. De Roos, N.M.; Schouten, G.; Katan, M.B. Yoghurt enriched with Lactobacillus acidophilus does not lower blood lipids in healthy men and women with normal to borderline high serum cholesterol levels. Eur. J. Clin. Nutr. 1999, 53, 277-280. [CrossRef]

13. Sergeev, I.N.; Aljutaily, T.; Walton, G.; Huarte, E. Effects of Synbiotic Supplement on Human Gut Microbiota, Body Composition and Weight Loss in Obesity. Nutrients 2020, 12, 222. [CrossRef] [PubMed]

14. Ferrario, C.; Taverniti, V.; Milani, C.; Fiore, W.; Laureati, M.; De Noni, I.; Stuknyte, M.; Chouaia, B.; Riso, P.; Guglielmetti, S. Modulation of Fecal Clostridiales Bacteria and Butyrate by Probiotic Intervention with Lactobacillus paracasei DG Varies among Healthy Adults. J. Nutr. 2014, 144, 1787-1796. [CrossRef] [PubMed]

15. Neto, J.V.; de Melo, C.M.; Ribeiro, S.M.L. Effects of Three-Month Intake of Synbiotic on Inflammation and Body Composition in the Elderly: A Pilot Study. Nutrients 2013, 5, 1276-1286. [CrossRef] [PubMed]

16. Turroni, F.; Ventura, M.; Butto, L.F.; Duranti, S.; O'Toole, P.W.; Motherway, M.O.; van Sinderen, D. Molecular dialogue between the human gut microbiota and the host: Lactobacillus and Bifidobacterium perspective. Cell. Mol. Life Sci. 2014, 71, 183-203. [CrossRef]

17. Collins, M.D.; Gibson, G.R. Probiotics, prebiotics, and synbiotics: Approaches for modulating the microbial ecology of the gut. Am. J. Clin. Nutr. 1999, 69, 1052S-1057S. [CrossRef]

18. Neish, A.S. Microbes in gastrointestinal health and disease. Gastroenterology 2009, 136, 65-80. [CrossRef]

19. Crittenden, R.; Laitila, A.; Forssell, P.; Matto, J.; Saarela, M.; Mattila-Sandholm, T.; Myllarinen, P. Adhesion of Bifidobacteria to granular starch and its implications in probiotic technologies. Appl. Environ. Microbiol. 2001, 67, 3469-3475. [CrossRef]

20. Markowiak, P.; Slizewska, K. Effects of Probiotics, Prebiotics, and Synbiotics on Human Health. Nutrients 2017, 9, 1021. [CrossRef]

21. Worthley, D.L.; Le Leu, R.K.; Whitehall, V.L.; Conlon, M.; Christophersen, C.; Belobrajdic, D.; Mallitt, K.A.; Hu, Y.; Irahara, N.; Ogino, S.; et al. A human, double-blind, placebo-controlled, crossover trial of prebiotic, probiotic, and synbiotic supplementation: Effects on luminal, inflammatory, epigenetic, and epithelial biomarkers of colorectal cancer. Am. J. Clin. Nutr. 2009, 90, 578-586. [CrossRef]

22. Bartosch, S.; Woodmansey, E.J.; Paterson, J.C.; McMurdo, M.E.; Macfarlane, G.T. Microbiological effects of consuming a synbiotic containing Bifidobacterium bifidum, Bifidobacterium lactis, and oligofructose in elderly persons, determined by real-time polymerase chain reaction and counting of viable bacteria. Clin. Infect. Dis. 2005, 40, 28-37. [CrossRef]

23. Macfarlane, S.; Cleary, S.; Bahrami, B.; Reynolds, N.; Macfarlane, G.T. Synbiotic consumption changes the metabolism and composition of the gut microbiota in older people and modifies inflammatory processes: A randomised, double-blind, placebo-controlled crossover study. Aliment. Pharmacol. Ther. 2013, 38, 804-816. [CrossRef] [PubMed]

24. Min, Y.W.; Park, S.U.; Jang, Y.S.; Kim, Y.H.; Rhee, P.L.; Ko, S.H.; Joo, N.; Kim, S.I.; Kim, C.H.; Chang, D.K. Effect of composite yogurt enriched with acacia fiber and Bifidobacterium lactis. World J. Gastroenterol. 2012, 18, 4563-4569. [CrossRef] [PubMed]

25. Choi, S.C.; Kim, B.J.; Rhee, P.L.; Chang, D.K.; Son, H.J.; Kim, J.J.; Rhee, J.C.; Kim, S.I.; Han, Y.S.; Sim, K.H.; et al. Probiotic Fermented Milk Containing Dietary Fiber Has Additive Effects in IBS with Constipation Compared to Plain Probiotic Fermented Milk. Gut Liver 2011, 5, 22-28. [CrossRef] 
26. Shinde, T.; Perera, A.P.; Vemuri, R.; Gondalia, S.V.; Karpe, A.V.; Beale, D.J.; Shastri, S.; Southam, B.; Eri, R.; Stanley, R. Synbiotic Supplementation Containing Whole Plant Sugar Cane Fibre and Probiotic Spores Potentiates Protective Synergistic Effects in Mouse Model of IBD. Nutrients 2019, 11, 818. [CrossRef] [PubMed]

27. Bjorklund, M.; Ouwehand, A.C.; Forssten, S.D.; Nikkila, J.; Tiihonen, K.; Rautonen, N.; Lahtinen, S.J. Gut microbiota of healthy elderly NSAID users is selectively modified with the administration of Lactobacillus acidophilus NCFM and lactitol. Age 2012, 34, 987-999. [CrossRef] [PubMed]

28. Bedani, R.; Saad, S.M.I.; Sivieri, K. Potential benefits of probiotics, prebiotics, and synbiotics on the intestinal microbiota of the elderly. In Probiotics, Prebiotics, Synbiotics; Elsevier: Amsterdam, The Netherlands, 2016; pp. 525-538. [CrossRef]

29. Jy, K.; Ey, C. Changes in Korean Adult Females' Intestinal Microbiota Resulting from Kimchi Intake. J. Nutr. Food Sci. 2016, 6, 486. [CrossRef]

30. Blake, M.R.; Raker, J.M.; Whelan, K. Validity, and reliability of the Bristol Stool Form Scale in healthy adults and patients with diarrhea-predominant irritable bowel syndrome. Aliment. Pharmacol. Ther. 2016, 44, 693-703. [CrossRef]

31. Shima, T.; Amamoto, R.; Kaga, C.; Kado, Y.; Sasai, T.; Watanabe, O.; Shiinoki, J.; Iwazaki, K.; Shigemura, H.; Tsuji, H. Association of life habits and fermented milk intake with stool frequency, defecatory symptoms and intestinal microbiota in healthy Japanese adults. Benef. Microbes 2019, 10, 841-854. [CrossRef]

32. Pandey, K.R.; Naik, S.R.; Vakil, B.V. Probiotics, prebiotics and synbiotics-A review. J. Food Sci. Technol. 2015, 52, 7577-7587. [CrossRef]

33. Vuljanic, D.; Dojder, A.; Spoljaric, V.; Saracevic, A.; Dukic, L.; Krleza, J.L.; Tanaskovic, J.V.; Maradin, I.; Grzunov, A.; Vogrinc, Z.; et al. Analytical verification of 12 most commonly used urine dipsticks in Croatia: Comparability, repeatability, and accuracy. Biochem. Med. 2019, 29, 123-132. [CrossRef]

34. Lim, M.Y.; Song, E.J.; Kim, S.H.; Lee, J.; Nam, Y.D. Comparison of DNA extraction methods for human gut microbial community profiling. Syst. Appl. Microbiol. 2018, 41, 151-157. [CrossRef] [PubMed]

35. Dassi, E.; Ballarini, A.; Covello, G.; Quattrone, A.; Jousson, O.; De Sanctis, V.; Bertorelli, R.; Denti, M.A.; Segata, N. Enhanced microbial diversity in the saliva microbiome induced by short-term probiotic intake revealed by $16 \mathrm{~S}$ rRNA sequencing on the IonTorrent PGM platform. J. Biotechnol. 2014, 190, 30-39. [CrossRef] [PubMed]

36. Ogata, K.; Takeshita, T.; Shibata, Y.; Matsumi, R.; Kageyama, S.; Asakawa, M.; Yamashita, Y. Effect of coffee on the compositional shift of oral indigenous microbiota cultured in vitro. J. Oral Sci. 2019, 61, 418-424. [CrossRef] [PubMed]

37. Adamiak, J.; Otlewska, A.; Tafer, H.; Lopandic, K.; Gutarowska, B.; Sterflinger, K.; Pinar, G. First evaluation of the microbiome of built cultural heritage by using the Ion Torrent next generation sequencing platform. Int. Biodeterior. Biodegrad. 2018, 131, 11-18. [CrossRef]

38. Malapelle, U.; Vigliar, E.; Sgariglia, R.; Bellevicine, C.; Colarossi, L.; Vitale, D.; Pallante, P.; Troncone, G. Ion Torrent next-generation sequencing for routine identification of clinically relevant mutations in colorectal cancer patients. J. Clin. Pathol. 2015, 68, 64-68. [CrossRef] [PubMed]

39. Yoon, S.H.; Ha, S.M.; Kwon, S.; Lim, J.; Kim, Y.; Seo, H.; Chun, J. Introducing EzBioCloud: A taxonomically united database of $16 S$ rRNA gene sequences and whole-genome assemblies. Int. J. Syst. Evol. Microbiol. 2017, 67, 1613-1617. [CrossRef] [PubMed]

40. Dave, V.; Street, K.; Francis, S.; Bradman, A.; Riley, L.; Eskenazi, B.; Holland, N. Bacterial microbiome of breast milk and child saliva from low-income Mexican-American women and children. Pediatric Res. 2016, 79, 846-854. [CrossRef]

41. Afgan, E.; Baker, D.; Batut, B.; van den Beek, M.; Bouvier, D.; Cech, M.; Chilton, J.; Clements, D.; Coraor, N.; Gruning, B.A.; et al. The Galaxy platform for accessible, reproducible and collaborative biomedical analyses: 2018 update. Nucleic Acids Res. 2018, 46, W537-W544. [CrossRef]

42. Robles-Vera, I.; Callejo, M.; Ramos, R.; Duarte, J.; Perez-Vizcaino, F. Impact of Vitamin D Deficit on the Rat Gut Microbiome. Nutrients 2019, 11, 2564. [CrossRef]

43. Minamida, K.; Nishimura, M.; Miwa, K.; Nishihira, J. Effects of dietary fiber with Bacillus coagulans lilac-01 on bowel movement and fecal properties of healthy volunteers with a tendency for constipation. Biosci. Biotechnol. Biochem. 2015, 79, 300-306. [CrossRef]

44. Cancello, R.; Turroni, S. Effect of short-term dietary intervention and probiotic mix supplementation on the gut microbiota of elderly obese women. Nutrients 2019, 11, 3011. [CrossRef] [PubMed] 
45. Duncan, S.H.; Flint, H.J. Probiotics and prebiotics and health in ageing populations. Maturitas 2013, 75, 44-50. [CrossRef] [PubMed]

46. Landete, J.M.; Gaya, P.; Rodriguez, E.; Langa, S.; Peiroten, A.; Medina, M.; Arques, J.L. Probiotic Bacteria for Healthier Aging: Immunomodulation and Metabolism of Phytoestrogens. BioMed Res. Int. 2017, 2017. [CrossRef] [PubMed]

47. Jennings, A.; Tang, J.; Gillings, R.; Perfecto, A.; Dutton, J.; Speakman, J.; Fraser, W.D.; Nicoletti, C.; Berendsen, A.A.M.; de Groot, L.; et al. Changing from a Western to a Mediterranean-style diet does not affect iron or selenium status: Results of the New Dietary Strategies Addressing the Specific Needs of the Elderly Population for Healthy Aging in Europe (NU-AGE) 1-year randomized clinical trial in elderly Europeans. Am. J. Clin. Nutr. 2020, 111, 98-109.

48. Salazar, N.; Valdes-Varela, L.; Gonzalez, S.; Gueimonde, M.; de Los Reyes-Gavilan, C.G. Nutrition and the gut microbiome in the elderly. Gut Microbes 2017, 8, 82-97. [CrossRef]

49. Gill, H.S.; Rutherfurd, K.J.; Cross, M.L.; Gopal, P.K. Enhancement of immunity in the elderly by dietary supplementation with the probiotic Bifidobacterium lactis HN019. Am. J. Clin. Nutr. 2001, 74, 833-839. [CrossRef]

50. Salazar, N.; Arboleya, S.; Valdes, L.; Stanton, C.; Ross, P.; Ruiz, L.; Gueimonde, M.; de los Reyes-Gavilan, C.G. The human intestinal microbiome at extreme ages of life. Dietary intervention as a way to counteract alterations. Front. Genet. 2014, 5, 406. [CrossRef]

51. Kim, S.; Jazwinski, S.M. The Gut Microbiota and Healthy Aging: A Mini-Review. Gerontology 2018, 64, 513-520. [CrossRef]

52. O'Toole, P.W.; Claesson, M.J. Gut microbiota: Changes throughout the lifespan from infancy to elderly. Int. Dairy J. 2010, 20, 281-291. [CrossRef]

53. Silvi, S.; Verdenelli, M.C.; Orpianesi, C.; Cresci, A. EU project Crownalife: Functional foods, gut microflora and healthy ageing-Isolation and identification of Lactobacillus and Bifidobacterium strains from fecal samples of elderly subjects for a possible probiotic use in functional foods. J. Food Eng. 2003, 56, 195-200. [CrossRef]

54. Bouhnik, Y.; Achour, L.; Paineau, D.; Riottot, M.; Attar, A.; Bornet, F. Four-week short chain fructo-oligosaccharides ingestion leads to increasing fecal Bifidobacteria and cholesterol excretion in healthy elderly volunteers. Nutr. J. 2007, 6, 42. [CrossRef]

55. Lahtinen, S.J.; Tammela, L.; Korpela, J.; Parhiala, R.; Ahokoski, H.; Mykkanen, H.; Salminen, S.J. Probiotics modulate the Bifidobacterium microbiota of elderly nursing home residents. Age 2009, 31, 59-66. [CrossRef] [PubMed]

56. Selma, M.V.; Beltran, D.; Luna, M.C.; Romo-Vaquero, M.; Garcia-Villalba, R.; Mira, A.; Espin, J.C.; Tomas-Barberan, F.A. Isolation of Human Intestinal Bacteria Capable of Producing the Bioactive Metabolite Isourolithin A from Ellagic Acid. Front. Microbiol. 2017, 8, 1521. [CrossRef] [PubMed]

57. Romo-Vaquero, M.R.-V.; Cortés-Martín, A.; Loria-Kohen, V.; De Molina, A.R.; García-Mantrana, I.; Collado, M.C.; Espín, J.C.; Selma, M.V. Deciphering the Human Gut Microbiome of Urolithin Metabotypes: Association with Enterotypes and Potential Cardiometabolic Health Implications. Mol. Nutr. Food Res. 2018, 63. [CrossRef]

58. Cortés-Martín, A.; Romo-Vaquero, M.; García-Mantrana, I.; Rodríguez-Varela, A.; Collado, M.C.; Espín, J.C.; Selma, M.V. Urolithin Metabotypes can Anticipate the Different Restoration of the Gut Microbiota and Anthropometric Profiles during the First Year Postpartum. Nutrients 2019, 11, 2079. [CrossRef] [PubMed]

59. Tomas-Barberan, F.A.; Garcia-Villalba, R.; Gonzalez-Sarrias, A.; Selma, M.V.; Espin, J.C. Ellagic Acid Metabolism by Human Gut Microbiota: Consistent Observation of Three Urolithin Phenotypes in Intervention Trials, Independent of Food Source, Age, and Health Status. J. Agric. Food Chem. 2014, 62, 6535-6538. [CrossRef] [PubMed]

60. Tian, S.Y.; Wang, J.; Yu, H.; Wang, J.; Zhu, W.Y. Changes in Ileal Microbial Composition and Microbial Metabolism by an Early-Life Galacto-Oligosaccharides Intervention in a Neonatal Porcine Model. Nutrients 2019, 11, 1753. [CrossRef]

61. Kameyama, K.; Itoii, K. Intestinal Colonization by a Lachnospiraceae Bacterium Contributes to the Development of Diabetes in Obese Mice. Microbes Environ. 2014, 29, 427-430. [CrossRef] [PubMed]

62. Chen, J.Z.; Thomsen, M.; Vitetta, L. Interaction of gut microbiota with dysregulation of bile acids in the pathogenesis of nonalcoholic fatty liver disease and potential therapeutic implications of probiotics. J. Cell. Biochem. 2019, 120, 2713-2720. [CrossRef] 
63. Guirong, Y.E.; Minjie, Z.; Lixin, Y.U.; Junsheng, Y.E.; Lin, Y.; Lisha, S. Gut microbiota in renal transplant recipients, patients with chronic kidney disease and healthy subjects. J. South. Med Univ. 2018, 38, 1401-1408. [CrossRef]

64. Vacca, M.; Celano, G.; Calabrese, F.M.; Portincasa, P.; Gobbetti, M.; De Angelis, M. The Controversial Role of Human Gut Lachnospiraceae. Microorganisms 2020, 8, 573. [CrossRef]

65. Wang, F.Q.; Zhang, P.; Jiang, H.L.; Cheng, S.L. Gut Bacterial Translocation Contributes to Microinflammation in Experimental Uremia. Dig. Dis. Sci. 2012, 57, 2856-2862. [CrossRef] [PubMed]

66. De Angelis, M.; Montemurno, E.; Piccolo, M.; Vannini, L.; Lauriero, G.; Maranzano, V.; Gozzi, G.; Serrazanetti, D.; Dalfino, G.; Gobbetti, M.; et al. Microbiota and Metabolome Associated with Immunoglobulin A Nephropathy (IgAN). PLoS ONE 2014, 9, e99006. [CrossRef] [PubMed]

67. Vaziri, N.D.; Wong, J.; Pahl, M.; Piceno, Y.M.; Yuan, J.; DeSantis, T.Z.; Ni, Z.M.; Nguyen, T.H.; Andersen, G.L. Chronic kidney disease alters intestinal microbial flora. Kidney Int. 2013, 83, 308-315. [CrossRef] [PubMed]

68. Laitinen, K.; Poussa, T.; Isolauri, E.; Nutrition, A.M.I.; Intestinal Microbiota, G. Probiotics and dietary counselling contribute to glucose regulation during and after pregnancy: A randomised controlled trial. Br. J. Nutr. 2009, 101, 1679-1687. [CrossRef]

69. Ranganathan, N.; Friedman, E.A.; Tam, P.; Rao, V.; Ranganathan, P.; Dheer, R. Probiotic dietary supplementation in patients with stage 3 and 4 chronic kidney disease: A 6-month pilot scale trial in Canada. Curr. Med Res. Opin. 2009, 25, 1919-1930. [CrossRef]

70. Firouzi, S.; Mohd-Yusof, B.N.; Majid, H.A.; Ismail, A.; Kamaruddin, N.A. Effect of microbial cell preparation on renal profile and liver function among type 2 diabetics: A randomized controlled trial. BMC Complem. Altern. Med. 2015, 15, 1-10. [CrossRef]

71. Cirit, M.; Toprak, O.; Yesil, M.; Bayata, S.; Postaci, N.; Pupim, L.; Esi, E. Angiotensin-converting enzyme inhibitors as a risk factor for contrast-induced nephropathy. Nephron Clin. Pract. 2006, 104, C20-C27. [CrossRef]

72. Kalliomaki, M.; Salminen, S.; Poussa, T.; Isolauri, E. Probiotics during the first 7 years of life: A cumulative risk reduction of eczema in a randomized, placebo-controlled trial. J. Allergy Clin. Immunol. 2007, 119, 1019-1021. [CrossRef]

73. Sugiura, S.H.; Hardy, R.W.; Roberts, R.J. The pathology of phosphorus deficiency in fish-A review. J. Fish Dis. 2004, 27, 255-265. [CrossRef]

74. Lee, Y.; Lee, H.J.; Lee, H.S.; Jang, Y.A.; Kim, C.I. Analytical dietary fiber database for the National Health and Nutrition Survey in Korea. J. Food Compos. Anal. 2008, 21, S35-S42. [CrossRef]

75. Kim, S.H.; Kim, M.S.; Lee, M.S.; Park, Y.S.; Lee, H.J.; Kang, S.-A.; Lee, H.S.; Lee, K.-E.; Yang, H.J.; Kim, M.J.; et al. Korean diet: Characteristics and historical background. J. Ethn. Foods 2016, 3, 26-31. [CrossRef]

76. Shin, J.H.; Jung, S.; Kim, S.A.; Kang, M.S.; Kim, M.S.; Joung, H.; Hwang, G.S.; Shin, D.M. Differential Effects of Typical Korean Versus American-Style Diets on Gut Microbial Composition and Metabolic Profile in Healthy Overweight Koreans: A Randomized Crossover Trial. Nutrients 2019, 11, 2450. [CrossRef] [PubMed]

(C) 2020 by the authors. Licensee MDPI, Basel, Switzerland. This article is an open access article distributed under the terms and conditions of the Creative Commons Attribution (CC BY) license (http://creativecommons.org/licenses/by/4.0/). 